\title{
Aplicativos de tradução para Libras e a busca pela validade social da Tecnologia Assistiva
}

\section{Ygor Corrêa $^{1}$, Maristela Compagnoni Vieira ${ }^{1}$, Lucila Maria Costi Santarosa ${ }^{1}$, Maria Cristina Villanova Biasuz ${ }^{1}$}

\author{
${ }^{1}$ Programa de Pós-Graduação em Informática na Educação - Universidade Federal do \\ Rio Grande do Sul (UFRGS). Porto Alegre - RS - Brasil \\ correaygorprofegmail.com, maricompagnoniegmail.com, \\ lucila.santarosa@ufrgs.br, mcbiazuseufrgs.br
}

\begin{abstract}
This paper presents HandTalk and ProDeaf apps, which are automatic translators of Portuguese into Brazilian Sign Language (Libras) and then analyzes one of them from three perspectives: the multimediatic, the multimodality and the socialization of deaf people. While the use of the app seeks an inclusive technology strategy, we attempted to identify, in narratives of deaf and hearing people, originated from a teachers' training course about assistive technologies, discursive fragments from this perspective. The data collected showed that, although ProDeaf app presents limitations in its digital interface, using dactylology or manifesting the absence of some signs, it can promote the learning of the languages involved, aspect that gives it social, political and cultural validity.
\end{abstract}

Resumo. Este artigo apresenta os aplicativos HandTalk e ProDeaf, os quais são tradutores automáticos de Língua Portuguesa para a Lingua Brasileira de Sinais (Libras) e, em seguida, analisa um deles sob três perspectivas: a multimidiática, a multimodalidade e quanto à socialização do sujeito surdo. Conquanto que a utilização do aplicativo visa uma estratégia tecnológica inclusiva, buscou-se identificar, em narrativas de sujeitos surdos e ouvintes, oriundas de um curso de formação de professoresem tecnologias assistivas, fragmentos discursivos nesta perspectiva. Os dados coletados evidenciaram que, embora o aplicativo ProDeaf apresente limitações em sua interface digital, manifestando a inexistência de alguns sinais, esse pode potencializar a aprendizagem das línguas envolvidas, aspecto que lhe confere validade social, política e cultural.

\section{Introdução}

Em se tratando de sujeitos com deficiência auditiva, muito já fora discutido quanto a submetê-los a um aprendizado induzido à Língua Portuguesa [Quadros, 2003; Sá, 2011], sendo essa atualmente reconhecida como segunda língua, dado o consenso contemporâneo acerca da utilização da Língua Brasileira de Sinais (Libras) como primeira língua entre os sujeitos surdos. Entretanto, tendo em vista que os sujeitos surdos, muitas vezes, acabam por serem inseridos em contextos formais e informais de uso da Língua Portuguesa, esses se deparam com diversas barreiras comunicativas. $\mathrm{O}$ que significa que o estabelecimento de um contrato comunicacional oralizado fica silenciado socialmente, tanto por parte do surdo, que se sente excluído, como quanto ao ouvinte, que não tem domínio de Libras. 
Diante disso, este estudo adota construtos sociointeracionistas, segundo os quais o aprendizado humano pressupõe uma natureza social específica, por meio de um processo que permite aos sujeitos penetrar na vida intelectual daqueles que os cercam [Vygotsky, 1989]. Destaca-se assim a linguagem como uma das principais ferramentas para o domínio dos meios sociais, ou seja, o crescimento intelectual do sujeito. No que diz respeito ao desenvolvimento do sujeito surdo, Vygotsky apresenta a surdez como uma deficiência social, por excelência [Vygotsky, 2010], e que, embora seja mais adequado ao sujeito surdo a comunicação por meio da língua de sinais, essa seria um fator limitador da condição social destas pessoas, uma vez que insere o sujeito apenas no restrito círculo daqueles que a conhecem. Referente a isso, Vygotsky (2010) aponta como deficiente o contexto social que não atende à demanda do sujeito que apresenta alguma alteração biológica frente as suas necessidades cotidianas. Assim, não é a deficiência que se mostra restritiva, mas a inequidade das estruturas sociais.

Para Quadros (2003) o contexto social que favorece a pessoa surda é aquele em que ela se realiza plenamente em suas interações linguísticas. Diante dessa problemática, este artigo, em um primeiro momento, apresenta os aplicativos HandTalk e ProDeaf Móvel, ambos elaborados a partir de pesquisas recentes realizadas em universidades do nordeste brasileiro - Universidade Federal de Pernambuco (ProDeaf Móvel) e Universidade Federal do Alagoas (HandTalk) - os quais são gratuitos e realizam traduções automáticas de palavras, termos e pequenas frases da Língua Portuguesa para Libras, por meio de um avatar animado. Então, na medida em que esses aplicativos enquadram-se no escopo das Tecnologias Assistivas (TAs) [Lima e Santarosa, 2003], por possivelmente desempenharem o papel de mediadores de interações linguísticas, torna-se relevante analisar suas funcionalidades, a partir de narrativas empíricas, quando da operacionalização de processos comunicativos entre sujeitos surdos e ouvintes.

Após essa abordagem, descreve-se a interface do aplicativo ProDeaf Móvel, quanto aos recursos multimodais e multimidiáticos que esse comporta, com vistas a verificar sua validade social enquanto TA. Desta forma, o presente artigo está dividido em: 1.Introdução; 2.A inclusão da pessoa surda mediada pela tecnologia; 3.Multimodalidade e Multimídia; 4.0 estudo realizado; 5.Análise de dados; 6.Considerações finais; e Referências.

\section{A inclusão da pessoa surda mediada pela tecnologia}

Nesta seção compreende que, embora a proposta educacional inclusiva, relacionada à pessoa surda, seja a do bilinguismo [Brasil, 2008], ainda existem escolas comuns que não apresentam estrutura educacional capaz de permitir ao surdo interações sociais efetivas por meio de sua língua materna, a Libras [Quadros, 2003]. Deste modo, inferese que enquanto espaço de desenvolvimento e socialização, a escola deve proporcionar processos de construção de autonomia e de subjetivação, os quais constituem os sujeitos. De acordo com Touraine (2009) e Bakhtin (1990) é por meio desses processos que se consolida a construção, por parte do indivíduo ou do grupo, de si mesmo enquanto sujeito.

Diante desta perspectiva, a problematização das representações sociais ajuda a romper a barreira dos preconceitos e estabelece novas estruturas interacionais. Neste tocante, inserir a pessoa surda via tecnologia pode ser uma das formas de promover sua sociabilização, potencializando processos de interação, comunicação e subjetivação. 
Então, visando corroborar o papel das tecnologias na vida e na constituição dos sujeitos, este estudo apoia-se em uma inferência de Lévy (2000), que afirma que a tecnologia pode contribuir para a superação de uma situação de inferioridade, desde que os sujeitos estejam em condições de participar ativamente dos processos de inteligência coletiva, ao passo que a luta pela inclusão deve visar o ganho de autonomia dos grupos excluídos.

Para tanto, parte-se da premissa de que toda a atividade humana é mediada por ferramentas, sendo essas gradualmente incorporadas e que, por sua vez, alteram as práticas interacionais dos sujeitos. Diante disso, segundo Warschauer $(2006$, p.153) "as ferramentas não apenas facilitam a ação que poderia ter ocorrido sem elas, mas, ao serem incluídas no processo comportamental, alteram o fluxo e a estrutura das funções mentais" como é o caso de pessoas surdas. Ainda na perspectiva vygotskyana, adota-se o conceito de Nível de Desenvolvimento Real (NDR), em que se encontram os saberes e as vivências prévias do sujeito, que podem ser transformados quando da utilização de uma ferramenta tecnológica, de modo a enriquecer os processos interacionais. Desta maneira, essas podem realizar o andaimento [Vygotsky, 2010] dos processos de desenvolvimento humano, logo, de aprendizagem, movendo os saberes do sujeito para a Zona de Desenvolvimento Proximal (ZDP).

Portanto, é na ZDP que compreende-se que o desenvolvimento das capacidades cognitivas, por meio de interações significativas, pode ocasionar a aprendizagem de novos conhecimentos ou, então aprimorar os já existentes. Na busca por TAs [Cook e Hussey, 1995; Ramires et al, 2013], potencialmente mediadoras da construção de conhecimento, de processos de subjetivação e de autonomia, identificou-se no ano de 2013, o surgimento dos aplicativos HandTalk e ProDeaf Móvel no mercado brasileiro de aplicativos para dispositivos móveis. Uma vez que ambos os aplicativos, outrora mencionados, são voltados para a inclusão social e comportam recursos digitais multimodais e multimídia, mostra-se pertinente analisá-los sob a ótica de sujeitos surdos e ouvintes.

\section{Multimodalidade e Multimídia}

As Tecnologias de Informação e Comunicação (TICs), no cenário digital de cibercultura [Lévy, 2000], reúnem diversos conteúdos e, para isso, utilizam-se de diferentes modos de apresentação das informações, as quais visam transmitir. Essas, por sua vez, visamoferecer estratégias condizentes com diferentes estilos de aprendizagem, o que significa que há uma preocupação acerca do usuário. Esse pressuposto também é adotado pelas TAs [Lima e Santarosa, 2003], como referido nas Seções 1 e 2. Desta maneira, o design instrucional e/ou interface [Gagné, 2005; Moreno e Mayer, 2007] de um objeto de aprendizagem ou aplicativo, de modo geral, é composto por elementos multimodais e multimidiáticos. O conceito de multimodalidade [Moreno e Mayer, 2007; Low e Sweller, 2005] define que um determinado conteúdo pode ser melhor absorvido quando representações verbais e não-verbais são combinadas em um recurso tecnológico mediativo que vise à aprendizagem. Em relação a esse conceito cabe distinguir modo de modalidade.

O modo é descrito como o código utilizado para representar a informação, podendo ser verbal (oral ou escrito) ou não-verbal (ilustrações, fotos, vídeos e animações). Já a modalidade é representada pelos sentidos receptores (auditivo ou visual) utilizados para receber a informação [Moreno e Mayer, 2007]. Ao ajustar a recepção do modo à 
modalidade, perante suas reais condições de aprendizagem e/ou limitações, o sujeito torna-se um "produtor de sentido" [Moreno e Mayer, 2007] quanto às escolhas que faz, visando seu próprio estilo de construção de conhecimento. Daí decorre a relevância de oferecer mais de um modo e modalidade. Essas escolhas podem ser, por exemplo, definir se quer: ouvir ou ler um texto; oralizar ou digitar aquilo que quer gravar e/ou converter; controlar a velocidade de um vídeo ou áudio, diante de seu ritmo de aprendizagem; e até mesmo encontrar espaços para notificar limitações técnicas em uma plataforma educacional, sotfware ou aplicativo, quanto à ação que está realizando, como forma de registrá-las para o conhecimento de programadores, professores, tutores e/ou desenvolvedores.

Da complexa combinação entre modos e modalidades também surge a necessidade de que estas sejam utilizadas com cautela em um recurso digital, de forma a evitar a sobreposição dessas que, se acionadas, simultaneamente, podem desencadear possíveis processos de sobrecarga cognitiva [Moreno e Mayer, 2007; Sweller, Van Merrienboer e Paas, 1998], quanto à atividade em execução. Portanto, uma excessiva exposição simultânea às informações pode reduzir a absorção do conteúdo, assim como o interesse pela ferramenta. Posto isso, o conceito de aprendizagem em multimídia entra em cena, uma vez que esse é responsável por fornecer subsídios para que modos e modalidades, por intermédio de recursos digitais, possam ser incorporados a um objeto de aprendizagem e/ou um aplicativo [Moreno e Mayer, 2007]. A multimídia, assim, constitui-se como um sistema organizado, composto por múltiplas mídias aglomeradas com o objetivo de propiciar formas interativas de comunicação, a fim de transmitir conteúdos por meio de textos, animações, áudios e vídeos. Essas características serão tratadas na Seção 5. A seguir, apresenta-se o estudo realizado.

\section{$4 \mathrm{O}$ estudo realizado}

Esta é uma pesquisa qualitativa [Moraes, 2003] que analisa a utilização dos aplicativos ProDeaf Móvel e HandTalk, a partir do viés sociointeracionista [Vygotsky, 2010], por meio da relação dialógica [Bakhtin, 1990] constituída entre sujeitos surdos e ouvintes, tendo como mediador um tipo de processo inclusivo mediado pelo uso de TA. Os sujeitos dessa pesquisa foram os professores que realizaram o Curso de Formação Continuada de Professores em Tecnologias de Informação e Comunicação Acessíveis do Núcleo de Informática na Educação Especial (NIEE) da Universidade Federal do Rio Grande do Sul (UFRGS).

A formação é oferecida à distância por meio da plataforma Teleduc e já se encontra na $18^{\mathrm{a}}$ edição, tendo atendido cerca de 9.000 professores. Entre as propostas do curso está a utilização de TAs em atividades práticas no contexto da escola inclusiva, como é o caso dos aplicativos ProDeaf Móvel e HandTalk, cujas apreciações realizadas pelos professores, em parceria com sujeitos surdos e ouvintes, resultaram no corpus de análise deste estudo. O objetivo geral da atividade proposta no curso era não apenas apresentar os aplicativos para os professores cursistas, mas levá-los a uma reflexão que abarcasse os seguintes aspectos: a) descrição do sujeito convidado a participar da interação, b) relato da realização da atividade e c) opinião dos sujeitos com relação às potencialidades e às fragilidades das ferramentas.

O estudo foi composto por dois grupos de sujeitos, intitulados Grupo A e Grupo B. O Grupo A foi composto por 14 sujeitos (6 professores ouvintes, 6 deficientes auditivos e 
2 intérpretes de Libras), dando origem a 9 narrativas e 36 fragmentos discursivos. Já o Grupo B foi composto por 62 sujeitos (28 professores ouvintes, 29 deficientes auditivos e 5 intérpretes de Libras), originando 29 narrativas e 133 fragmentos discursivos. As narrativas resultantes das interações entre surdos e ouvintes quanto aos aplicativos, enquanto reflexões em caráter de inclusão social [Warschauer, 2006], aprendizagem [Vygotsky, 2010] e usabilidade [Nielsen, 2003] constituíram os dados coletados.

Quanto à análise das narrativas, optou-se pela estratégia metodológica proposta por Moraes (2003), que envolve a técnica de Análise de Conteúdo [Moraes, 2003; Bardin, 2007] e prevê a identificação da emergência de conceitos, a partir da incidência de unidades de sentido presentes nos discursos, autorizando o agrupamento dessas nas categorias apresentadas na (Figura 1). O desenvolvimento do estudo, composto pelos dois grupos, por sua vez, ocorreu em três etapas, sendo essas: Etapa 1 - analisar as características dos dois aplicativos; Etapa 2 - investigar nas narrativas dos sujeitos do Grupo A fragmentos discursivos quanto ao caráter tecnológico inclusivo de ambos os aplicativos; e Etapa 3 - delimitar a partir do aplicativo ProDeaf Móvel (dado o maior número de recursos - Tabela 1) fragmentos discursivos extraídos do Grupo $\mathrm{B}$ que, de fato, corroborassem o caráter social inclusivo do aplicativo, assim como sua constituição multimidiática e multimodal.

\section{Análise}

Na Etapa 1 elaborou-se uma tabela composta pelo panorama das características técnicooperacionais dos aplicativos HandTalk e ProDeaf Móvel, enquanto análise comparativa entre suas funcionalidades (conforme Tabela 1).

Tabela 1: Características das ferramentas ProDeaf Móvel e HandTalk

\begin{tabular}{|c|c|c|}
\hline & $\begin{array}{c}\text { ProDeaf } \\
\text { Móvel }\end{array}$ & HandTalk \\
\hline Disponível para Android & SIM & SIM \\
\hline Disponível para IOS & SIM & SIM \\
\hline Disponível para Windows Phone 8 & SIM & $\mathrm{NÃO}$ \\
\hline Traduz Língua Portuguesa para Libras a partir da entrada de texto & SIM & SIM \\
\hline Traduz Língua Portuguesa para Libras a partir da entrada de voz & SIM & SIM \\
\hline Traduz Língua Portuguesa para Libras a partir da entrada de fotografias de textos & $\mathrm{NA \tilde {O }}$ & SIM \\
\hline Disponibiliza dicionário de sinais & SIM & $\mathrm{NÃO}$ \\
\hline Funciona sem necessidade de acesso à Internet no momento da utilização & SIM & $\mathrm{NÃO}$ \\
\hline Permite girar o personagem para visualizar o sinal em diferentes ângulos & NÃO & SIM \\
\hline Possibilita fácil notificação acerca de algum erro identificado na sinalização & SIM & $\mathrm{NÃO}$ \\
\hline
\end{tabular}

Após a análise das funcionalidades apresentadas na Tabela 1, passou-se à Etapa 2, na qual investigou-se empiricamente nas narrativas dos sujeitos do Grupo A, fragmentos discursivos que ressaltassem o caráter tecnológico-inclusivo de ambos os aplicativos. Nessa etapa, a análise das narrativas apontou situações inclusivas em caráter de aprendizagem formal (escolar) e informal (social), tanto da Língua Portuguesa quanto da Libras.

Os fragmentos discursivos, derivados das narrativas, permitiram a identificação de implicações, denominadas como potencialidades e fragilidades (Figura 1). Referente aos fragmentos discursivos, seis categorias de potencialidades foram identificadas, sendo essas: (A) potencial inclusivo - facilitação da interação social entre surdos e ouvintes nas atividades cotidianas, como comunicar-se em um banco, escola, loja, etc.; (B) ampliação do arcabouço linguístico da Língua Portuguesa para o sujeito surdo; (C) 
ampliação do arcabouço linguístico da Libras para o sujeito ouvinte - no que diz respeito à utilização do dicionário presente nos aplicativos; (D) facilidade de uso; (E) ampliação da autonomia do sujeito surdo [Vygotsky, 2010] para interpretação da comunicação por meio de português escrito, pois boa parte das interações estabelecidas entre os sujeitos surdos necessitam da Língua Portuguesa; e (F) constituição íntima do sujeito surdo [Touraine, 2009; Melucci, 2004], que possibilita que esse adquira contornos mais individualizados quando o termo cujo significado deseja saber apresenta aspectos muito íntimos para serem compartilhados.

Já em relação às fragilidades, cinco categorias também foram identificadas, sendo essas: (G) dicionário de sinais restrito e a consequente prática do português sinalizado(datilologia); $(\mathrm{H})$ dificuldades técnicas quanto ao uso da ferramenta ou alguma de suas funcionalidades; e (I) ausência do parâmetro de expressão facial/corporal. A categoria $(\mathrm{J})$ chama a atenção para a restrição da potencialidade inclusiva dos aplicativos, pois caso os sujeitos não possuam um dispositivo móvel do tipo smartphone ou tablet, dado é o custo elevado deste tipo de aparelho, não terão condições de utilizá-lo. Referente à categoria $(K)$, os sujeitos destacam a sinalização de traduções incorretas.

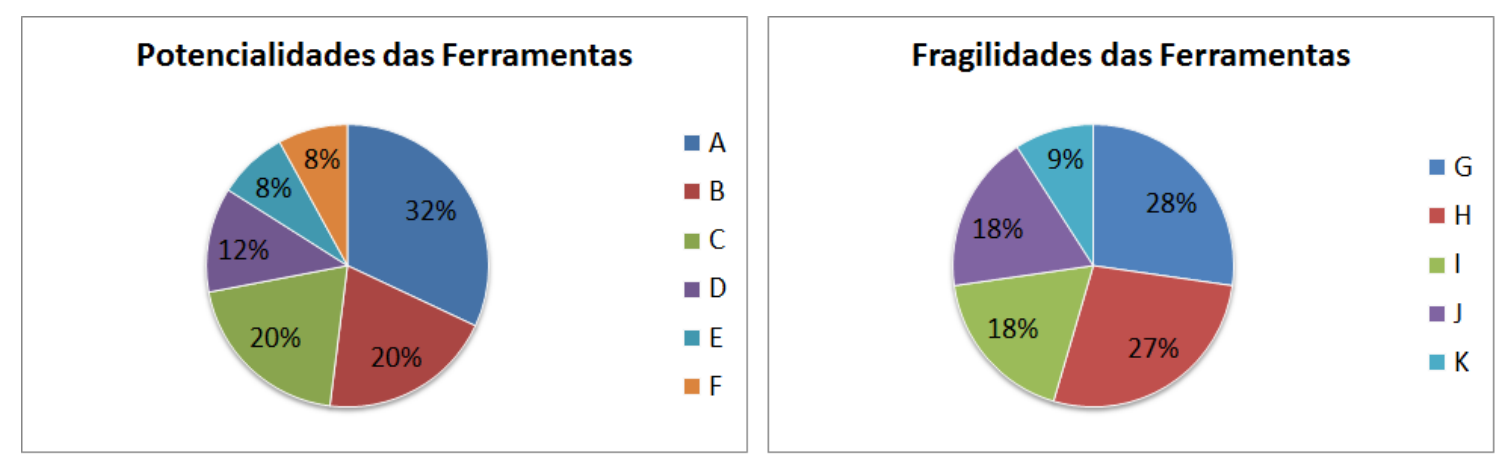

Figura 1: Potencialidades e fragilidades dos aplicativos a partir das narrativas

Concluídas as Etapas 1 e 2, passou-se à Etapa 3, visando o aprofundamento do caráter inclusivo social do aplicativo ProDeaf Móvel, uma vez que esse, quando submetido à investigação de seus recursos técnico-operacionais, sobressaiu-se em relação ao aplicativo HandTalk. Posto isso, com o Grupo B (composto por surdos e ouvintes), analisou-se a totalidade das narrativas desse grupo, porém extraindo fragmentos discursivos apenas de sujeitos surdos, os quais, de fato, corroborassem a referência inicial manifestada pelo Grupo A (composto por surdos e ouvintes), na medida em que, já neste grupo, tinha-se notado maior adesão ao aplicativo ProDeaf. Na Tabela 2, são apresentados exemplos de fragmentos discursivos, por parte de sujeitos surdos, os quais corroboram o carácter inclusivo do aplicativo ProDeaf Móvel frente às suas funcionalidades quando em contexto social, cultural e/ou escolar, como também manifestado pelo Grupo A, na Etapa 1.

Para além da análise investigativa das funcionalidades presentes no aplicativo ProDeaf Móvel (Tabela 1) e de narrativas que ressaltaram seu potencial incluviso (Grupo A e reforçadas pelo Grupo B - Tabela 2), identificou-se a necessidade de verificar sua constituição multimodal (tipos de código de entrada e de saída), assim como de que 
maneira esse operava quanto a seus recursos multimidiáticos (texto, imagem, áudio, vídeo).

Tabela 2: Depoimentos dos sujeitos

\begin{tabular}{l} 
Inclusão social, cultural e escolar \\
\hline “Através do aplicativo o aluno poderá pode ter acesso a Libras o tempo que desejar, aprimorando \\
assim a sua língua materna e consequentemente a sua segunda língua que também é muito \\
importante para seu convívio social." (Professora surda) \\
“O ProDeaf Móvel ajuda falar com minha família, minha avó escreve no papel eu olho no celular.” \\
(Estudante Surdo) \\
"Tem palavra que não sei no português e pesquiso no ProDeaf.” (Estudante Surdo) \\
"Quando uso o facebook ou amigo envia mensagem para o meu celular e não sei a palavra, eu digito \\
e vejo o sinal eu gosto.” (Estudante Surdo) \\
“Tem frase que é segredo não vou mostrar para ouvinte explicar para mim, o ProDeaf me ajuda.", \\
(Estudante Surdo) \\
“[..] esse aplicativo ajuda na comunicação com ouvintes que não conhece LS [Língua de Sinais] \\
então o ouvinte digita a palavra ou texto para se comunicar com surdos. Nas reuniões de trabalho \\
onde tem surdos e ouvintes: o surdo pensa "como vou acompanhar ou entender o que estão \\
discutindo?”, então esse tradutor ajuda. Quando surge na escola, no trabalho, na TV alguma \\
palavra desconhecida do Português, então pesquisam no ProDeaf Móvel.” (Estudante surdo)
\end{tabular}

No que se refere ao caráter multimodal do aplicativo ProDeaf Móvel, entendeu-se que esse reside na operação dos seguintes modos: a) visual (texto, imagem, vídeo) quando o sujeito pesquisado, na maioria das vezes, digita (a palavra, conceito ou frase) no teclado virtual e o avatar (animação em vídeo), media o processo, convertendo o dado informado para Libras; e b) sonoro (termo oralizado) quando o sujeito ouvinte/surdo, dependendo do grau de deficiência, opta por oralizar o termo, enquanto código de entrada, a ser convertido.

O avatar (vídeo) está, basicamente, em constante movimento, devido ao caráter linguístico-gestual inerente à Libras, que, por meio de expressões manuais e nãomanuais realiza a conversão solicitada. Ressalta-se, contudo, que o aplicativo não comporta saída de áudio, aspecto que se justifica devido à própria natureza da deficiência de seu usuário, muito embora, ao considerar que ouvintes façam uso deste, seria relevante o oferecimento de informação sonora, de forma optativa. Essa inferência abrange a noção de que trata-se de uma relação dialógica [Bakhtin, 1990], ou seja, bilateral, ao passo que se o ouvinte não estivesse no enquadre situacional comunicativo, logo o surdo não necessitaria de um aplicativo.

A análise dos recursos multimidiáticos presentes no aplicativo ProDeaf Móvel, os quais possibilitam que modos e modalidades [Moreno e Mayer, 2007] sejam acionados quando em situações reais de mediação de processos comunicativos derivou-se de testes realizados a partir das narrativas dos sujeitos do Grupo B. O aplicativo ProDeaf Móvel oferece ao usuário: a) o acesso a um dicionário; b) entrada de informação por meio de voz, c) entrada de informação por meio de texto e d) o acesso a outras opções. A Figura 2 apresenta a interface do aplicativo, na qual se pode observar, na Imagem A, os ícones referentes ao dicionário, entrada de voz, entrada de texto e acesso às configurações.

Os recursos multimídia foram identificados quanto à possibilidade de inserir: a) texto e/ou b) voz, opções essas que podem ser selecionadas por parte do usuário, permitindo 
que esse identifique a melhor forma de informar ao aplicativo o código de entrada (escrito ou oral) que deseja converter para Libras. Ambos os estilos de entrada operam distintamente, porém a captura de voz, por vezes, devido a ruídos periféricos, apresenta problemas quanto ao que é convertido. Assim, por meio de texto ou voz, ao receber o código de entrada, o avatar animado (vídeo, código de saída) passa ao fornecimento do sinal em Libras ou à realização de datilologia (quando da ausência do sinal), ação realizada por meio de gestos manuais e não-manuais ainda limitados.

$\mathrm{O}$ ícone referente às demais configurações (Figura 2, Imagem B) apresenta quatro opções: a) acesso ao tutorial de utilização da ferramenta simultaneamente em Libras e em Língua Portuguesa escrita, disponibilizada frente aos dois tipos de usuários, surdos e ouvintes; b) configurações e personalização, tais quais (i) status de conexão com a Internet, posto que a maior parte dos sinais está armazenada em nuvem; (ii) possibilidade de confirmação do reconhecimento de voz; (iii) atualização do arcabouço de sinais; (iv) atualização automática de sinais; e (v) velocidade da interpretação (Figura 2 - Imagem B). Outra possibilidade é a de contato com a comunidade virtual do aplicativo ProDeaf Móvel, simbolizada por um (I) (internet), que redireciona o usuário à rede social Facebook; assim como compartilha a homepage do aplicativo para a avaliação desse. Ainda dentre esses recursos é possível solicitar um novo sinal - ícone simbolizado por uma mão com o sinal de mais - que, ao ser selecionado, questiona se o usuário sentiu falta de algum sinal e exclama: "Solicita que a gente faz!". A funcionalidade é operacionalizada por meio da digitação, feita pelo usuário, quanto ao seu endereço de e-mail e relativa ao sinal a ser desenvolvido (Figura 2 - Imagem C).

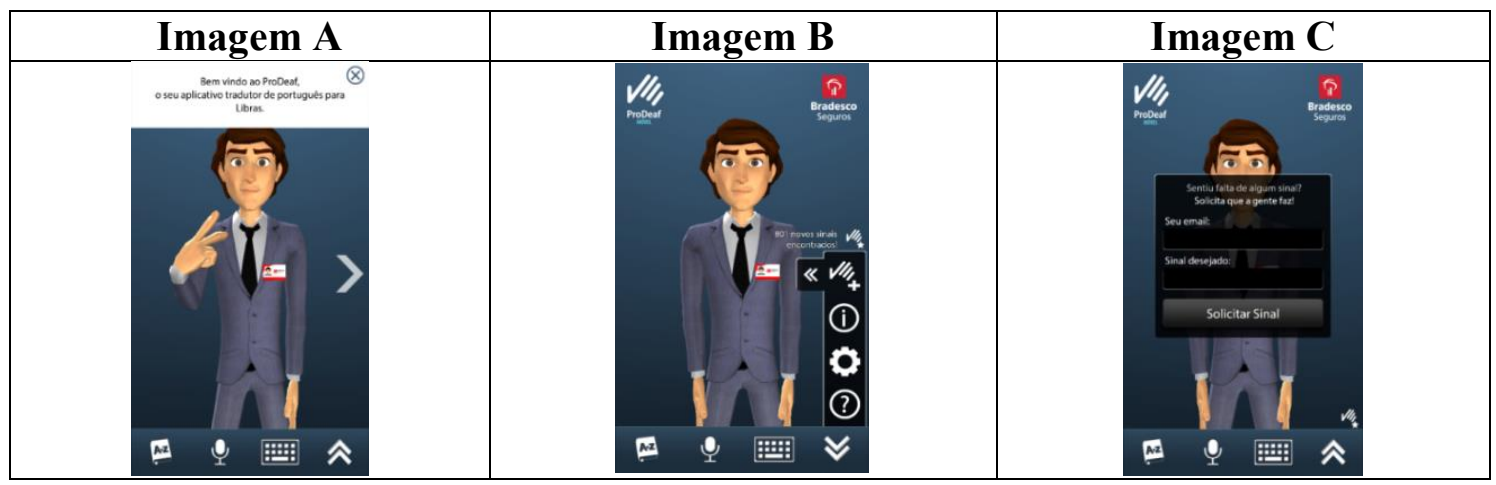

Figura 2: Interfaces das funcionalidades do aplicativo ProDeaf Móvel

\section{Considerações Finais}

Em síntese, quanto à análise geral das funcionalidades, observou-se a predominância de potencialidades em relação às fragilidades, o que evidencia o caráter mediativoinclusivo de ambos os aplicativos na relação comunicativa entre surdos e ouvintes. Aspecto este que reafirma a efetivação de processos de sociabilização dos sujeitos surdos e ouvintes de uma zona do conhecimento (NDR) para outra (ZDP) [Vygotsky, 2010], facilitando os processos interacionais e, consequentemente, contribuindo para a diminuição de processos de segregação social [Warschauer, 2006]. A promoção de processos de escolarização inclusivos [Quadros 2013], relativa aos aplicativos, foi constatada em diversos relatos, os quais manifestavam uma melhora significativa na comunicação entre sujeitos. Ao analisar narrativas que relatavam a concretização de uma comunicação efetiva entre pares, assim como no coletivo, o estudo apontou para a 
inclusão do sujeito surdo, evidenciando que esse passa a sentir-se inserido em sua sociedade de forma mais equânime, por meio das relações que estabelece de maneira mais autônoma apoiado pela TA.

No que se refere às fragilidades, acredita-se que essas devam-se ao recente lançamento dos aplicativos. Entretanto, esse problema tende a ser minimizado com a progressiva utilização das ferramentas, na medida em que os desenvolvedores possibilitam que a necessidade de implementação de novos sinais seja notificada. Constatou-se que permitir ao sujeito um diálogo com os desenvolvedores, via aplicativo, é relevante para a incorporação de nossos sinais.

Quanto à análise do caráter multimodal e multimidiático do aplicativo ProDeaf Móvel não observou-se a presença de sobrecarga cognitiva [Moreno e Mayer, 2007; Sweller, Van Merrienboer e Paas, 1998], pois o funcionamento dos recursos digitais ocorre em momentos distintos, uma vez que há um breve espaço de tempo entre a entrada do código informado e a saída do código fornecido. Por outro lado, o recurso de captura de voz, apresenta-se ainda problemático quanto à presença de ruídos periféricos, o que faz com que a conversão seja distorcida ou não realizada. Outro aspescto relevante é que na ausência de determinado sinal em Libras, o aplicativo utiliza-se de datilologia, o que não é totalmente aceito e/ou reconhecido pelos surdos [Secco e Silva, 2009].

O aplicativo ProDeaf Móvel mostrou cumprir principalmente com a função de sociabilização, tanto do surdo quanto do ouvinte, potencializando processos de aprendizagem das línguas envolvidas, aspecto que lhe confere validade social, política e cultural. Trabalhos futuros devem ser desenvolvidos no sentido de verificar com maior profundidade as fragilidades mencionadas pelos sujeitos em relação à ausência dos parâmetros não-manuais da Libras.

\section{Referências}

Bakhtin, M. (1990) Marxismo e filosofia da linguagem. Tradução Michel Lahud e Yara Frateschi Vieira. São Paulo: Hucitec.

Bardin, L. (2007) Análise de conteúdo. Lisboa: Ed. 70.

Brasil. (2008) Secretaria de Educação Especial. Politica Nacional de Educação Especial na Perspectiva da Educação Inclusiva. MEC/SEESP.

Cook, A.M. e Hussey, S. M. (1995) Assistive Technologies: Principles and Practices. St. Louis, Missouri. Mosby - Year Book, Inc.

Gagné, R.; Wagner, W.W.; Golas, K. C,; Keller, J.M. (2005) Principles of instructional design. 5 e. Thomson.

Lévy, P. (2000) Cibercultura. 2. Ed. São Paulo: Ed. 34.

Lima, C. R. U. de L.; Santarosa, L. M. C. (2003) Acessibilidade Tecnológica e Pedagógica na Apropriação das Tecnologias de Informação e Comunicação por Pessoas com Necessidades Educacionais Especiais. XIV Simpósio Brasileiro de Informática na Educação - NCE - IM/UFRJ.

Low, R., \& Sweller, J. (2005). The modality principle in multimedia learning. In: R. Mayer (Ed.), Cambridge handbook of multimedia learning (pp.147-158). New York: Cambridge University Press. 
Melucci, A. (2004) O jogo do eu: a mudança de si em uma sociedade global. São Leopoldo, RS: Editora Unisinos.

Moraes, R. (2003) Uma tempestade de luz: a compreensão possibilitada pela análise textual discursiva. Ciência Educação, Bauru, SP, v. 9, n. 2, p. 191-210.

Moreno, R.; Mayer R. (2007) Interactive multimodal learning environments. Educational Psychology Review, [n.1.], v. 19, p. 309-326.

Nielsen, J. (2003) Introduction to Usability. Alertbox: Current Issues in Web Usability. [S.I.:s.n.] Disponível em: <http://www.useit.com/alertbox/20030908.html>. Acesso em março de 2014.

Quadros, R. M. de. (2003) Situando as diferenças implicadas na educação de surdos: inclusão/exclusão. Ponto de Vista, Florianópolis, n.05, p. 81-111.

Ramires, L. O.; Souza, E.P.R.; Souza, J. N. de S.; Freire, M. R.; Januário, M. V.S.; Silva, R.E.F.; Gomes, R. S.F.; Lima, T.A.B. de. (2013) Como a Tecnologia Assistiva tem auxiliado o Processo de Ensino/Aprendizagem? Mapeamento Sistemático a partir dos Trabalhos Publicados no SBIE. II Congresso Brasileiro de Informática na Educação.

Sá, N. R. L. de. (2011) Surdos - qual escola?. Manaus: Editora Valer e Edua.

Secco, R. L.; Silva, M. H. L. F. da. (2009) Proposta de um Ambiente Interativo para Aprendizagem em Libras Gestual e Escrita. XX Simpósio Brasileiro de Informática na Educação.

Sweller, J.; Van Merrienboer, J.; Paas, F. (1998) Cognitive architecture and instructional design. Educational Psychology Review, 10, p. 251-296.

Touraine, A. (2009) Pensar outramente o discurso interpretativo dominante. Petrópolis, RJ: Vozes.

Vygotsky, L.S. (1989) Obras Completas: fundamentos da defectologia. Tomo V. Trad. Ma. del Carmen Ponce Fernández. Ciudad de La Habana: Editorial Pueblo y Educación.

Vygotsky, L.S. (2010) A formação social da mente. São Paulo: Martins Fontes, $4^{\mathrm{a}} \mathrm{ed}$.

Warschauer, M. (2006) Tecnologia e inclusão social: a exclusão digital em debate. São Paulo: Senac, 319 p. 